\title{
Experimental investigation of the effects of the presence and operation of tidal turbine arrays in a split tidal channel
}

\author{
Tim Daly ${ }^{1 *}$, Luke. E. Myers ${ }^{1}$, AbuBakr S. Bahaj ${ }^{1}$ \\ ${ }^{1}$ Sustainable Energy Research Group, University of Southampton, Southampton, United Kingdom \\ *Corresponding author. Tel: +44 2380592134,E-mail: td2e09@soton.ac.uk
}

\begin{abstract}
The installation of arrays and farms is the next major step in the development of tidal energy converters. Many tidal farms are currently in the process of development. A number of studies have also identified potentially lucrative sites for future farm and array development elsewhere. In some of these sites, the flow velocities can at least in part be attributed to the presence of constraining landmasses and the resultant splitting of channels into two or more sub channels. Given the cubic relationship between flow velocity and kinetic energy flux, even modest acceleration in these areas can cause a considerable increase the potential power available.

The analysis in this paper investigates flow acceleration effects in a split tidal channel due to the presence of tidal turbine arrays. As well as their presence, the effect of changing lateral and longitudinal position of the array and number of turbines in the array was also examined. Results show that flow acceleration of up to $14 \%$ can occur in an empty channel due to the presence of tidal arrays. This could potentially have major implications for tidal farm design in areas where channels branch into multiple sub channels.
\end{abstract}

Keywords: Split tidal channel, Obstruction, Actuator fences, Flow acceleration, Acoustic Doppler velocimeter.

\section{Introduction}

Studies have shown that the presence of tidal turbine arrays in any channel has the potential to have a significant effect of the surrounding flow environment [1]. They will also impact human activities such as shipping and sensitive environmental processes such as sediment transport, shore erosion and fish migration. Many areas worldwide in which split tidal channels are present have been identified as having high potential tidal energy resources. Examples include the Sound of Islay, Scotland, UK [2], Bay of Fundy, Canada [3] and Puget Sound, Washington, USA [4]. In areas such as these, aforementioned effects are likely to be greater, as placing turbines in one sub channel may alter the flow in some or all other sub channels. While many of these effects will obviously depend on the bathymetry of the site in question, there will undoubtedly be many generic effects which will be common to all sites of this kind.

This paper outlines the methods and results of experimentation carried out to examine some of the effects of tidal turbine arrays located in split tidal channels. Experimentation was carried out in the University of Southampton Chilworth hydraulics laboratory using a circulating water channel, actuator fences and acoustic Doppler velocimeters (ADV). The hypothetical site investigated was a simple channel which splits into two equally sized sub channels due to the presence of an impenetrable landmass between them. One of the sub channels had a tidal array installed, while the other was left empty. The resultant flow velocity in the empty sub channel was compared to the natural flow velocity to determine the percentage increase which resulted due to energy extraction.

\section{Review of previous studies}

Despite the high velocity magnitudes present in areas of split tidal channels, there is considerable variability in the bathymetry and hydrodynamic environment from site to site. This makes these areas much more complex to analyse from a resource assessment and environmental impact point of view. Also unlike single channel areas, it can be very difficult 
to determine theoretical expressions which will be applicable to all sites. One study which aimed to develop such theoretical expressions is outlined in [5]. The paper presents the case of a simple channel connected to two infinite oceans, and divided into two equal sub channels by an island in the centre. One sub channel has energy extracted by tidal energy converters, while the other is left empty. The authors derive expressions for power extracted by turbines and total fluid power as functions of head drop across the turbines and head drop across the entire channel between the two oceans respectively. The authors conclude that a maximum of $38.49 \%$ of total fluid power can be extracted, a figure which agrees with estimates for efficiency developed from single channel extraction theory developed by Garrett and Cummins in [6].

A site specific analysis of an area known as Johnstone Strait was carried out in [7]. The Johnstone Strait region consists of a number of sub channels, and the authors used both analytical methods developed by Garrett and Cummins [6] and a numerical model to examine the maximum power extracted by turbines in a total of four different sub channels. The authors found that their numerical model agreed reasonably well with the analytical theory developed in [8] for two particular cases. However for the other two investigated instances, theory was not valid, as the theory developed is only valid for instances where the flow of water cannot be diverted away from the sub channels where tidal turbines are installed [7]. Further analysis also examined some effects to the hydrodynamic environment by comparing natural tidal heights and amplitudes with those observed following energy extraction.

These studies use different methods of accounting for the presence of turbines, with [5] using head loss coefficients to calculate head drops and resultant power values, and [7] increasing natural bottom friction coefficient to include the effects of turbines presence. However undoubtedly the biggest difference is that [7] acknowledges the effects of energy extraction in different areas on the surrounding area, and attempts to quantify it briefly by examining changes to tidal amplitude and height. While [5] estimates high possible extractable power for an area of Johnstone Strait, there is no way for a potential developer to determine whether the changes to the surrounding hydrodynamic environment will render extraction of this energy unacceptable from an environmental point of view.

In contrast to both of these studies, the far field effects of tidal energy extraction was the sole subject of investigation in an analysis of four different tidal site configurations carried out in [8]. The four types of channel networks investigated were:

(a): A single constriction, which is a simple narrowing of a tidal channel.

(b): A multiply connected network, where flow is diverted from one channel into two sub channels, each of which contains a single constriction, and which meet again later in the flow.

(c): A branching network, where flow is diverted from one channel into two sub channels each of which contains a constriction. However these sub channels do not meet later in the flow.

(d): Serial constrictions, where a single channel contains a number of areas where constrictions are present.

Analytical methods were used to examine far field effects included shallow water equations, the conservation of mass and the conservation of energy. Results found that the largest tidal amplitude changes occur in the Branching network, biggest changes in kinetic power density occurring in the multiply connected network and changes to transport amplitude and frictional power dissipation approximately equal in all cases. 
These three investigations all contain elements of resource assessment which are crucial to the process of designing and installing a tidal turbine farm. However all rely solely on analytical and numerical models and no experimental results are present in these analyses. Section 3 outlines the methodology and reasoning for experimentation carried out to examine the changes to the hydrodynamic environment of a network of channels due to the presence of tidal energy converters. It is hoped that this analysis will further aid in the exploitation of the maximum potential energy in these sites with minimal and justifiable environmental effects.

\section{Experimental method}

\subsection{Flume setup}

The experimentation for this investigation was carried out in the indoor flume of the University of Southampton Chilworth hydraulics laboratory. The flume is a conventional gravity fed flume, with water pumped from sumps and through a flow channel, and mass flow rate, depth and flow velocity magnitude controlled via valves and a tail gate. The working section is $21 \mathrm{~m}$ in length, $1.37 \mathrm{~m}$ wide with a maximum flow depth of $0.5 \mathrm{~m}$. A $100 \mathrm{~mm}$ wide dividing wall was placed along the streamwise centerline of the flume over a length of $4 \mathrm{~m}$ (Fig. 1). The wall was placed between $8.5 \mathrm{~m}$ and $12.5 \mathrm{~m}$ from the inlet of the $21 \mathrm{~m}$ channel. This split the flow into two hypothetical sub channels in a similar fashion to an impenetrable landmass in a real tidal channel.

\subsection{Turbine array simulation}

Tidal turbine arrays were represented in the Chilworth flume using porous actuator fences. The porous actuator fence is a convenient alternative to rotating turbines in the analysis of tidal farms and arrays. The main difference between fences and turbines is opposed to extracting kinetic energy from a fluid, actuator fences convert this energy to small scale turbulence in their wake. Other differences include the inability of fences to induce swirl effects in the flow and the differences in the structure of vortices shed from both. These factors mean that actuator fences are unsuitable for examining farm or array power output or the structure of the near wake of tidal arrays.

However analysis in [9] has shown that they are highly accurate in predicting the far wake effects of tidal turbine farms, which are likely to impact on farm layout and surrounding flow environment. Actuator fences also have the advantage of being easier and cheaper to construct than turbines. They are also advantageous for numerical modeling, as CFD simulations with actuator fences can be run in steady state as opposed to unsteady state for rotating turbines, and also require much less complex meshes.

Two actuator fences were used in this analysis. They were created from $300 \mathrm{~mm}$ wide and $100 \mathrm{~mm}$ high sheets of PVC with a thickness of $4 \mathrm{~mm}$. Holes were drilled in the sheets to achieve the desired open to total area ratio's (porosities).

\subsection{Flow velocity magnitude measurements}

All flow velocity measurements were taken using an acoustic Doppler velocimeter (ADV). The instrument used for this work was set to sample at $50 \mathrm{~Hz}$, just below the noise floor. The sample volume is cylindrical with a fixed diameter of $6 \mathrm{~mm}$. the volume height is user-defined and was chosen to be $3 \mathrm{~mm}$. Larger sample volumes will intercept more suspended matter in the water leading to stronger acoustic return signals and greater accuracy. However this can be negated as velocity shear between the top and bottom of the sample volume can lead to inaccuracies. Due to the high levels of suspended matter in the Chilworth flume, no doubt arising from being located in a hard water area, the signal strengths were found to be very 
strong. With the water depth set to $0.3 \mathrm{~m}$ the sample height represented $1 \%$ of the depth thus ensuring that velocity shear was minimal across measurement volume. To eliminate any errors which may have occurred due to random velocity fluctuations, the data were filtered subsequent to experimentation. The method used in this instance was the velocity correlation filter outlined in [10]. Other filtering methods determine the criteria upon whether a measurement is invalid on the basis of the relationship between successive measurements. These methods would be unsuitable for this analysis, as groups of random fluctuations are likely to exist due to the turbulent nature of the flow downstream of the actuator fences. The velocity correlation filter is more suitable to the present study as the criteria for determining the validity of a measurement is calculated based on its relationship to all velocity measurements in the sample.

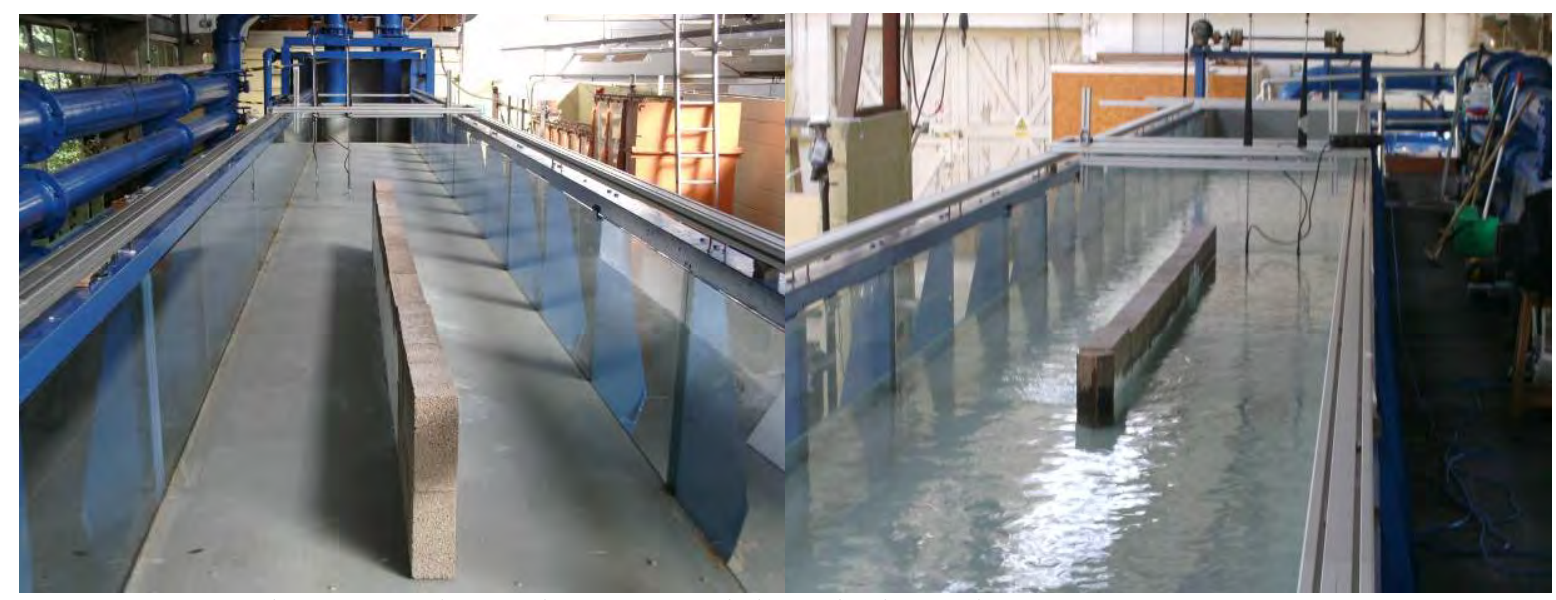

Fig.1. Front and rear of split mechanism in Chilworth channel.

\subsection{Base flow map and actuator fence positioning}

\subsubsection{Analysis of natural flow environment}

Initial flow mapping work was conducted in the absence of actuator fences in order to quantify the baseline flow environment with the split present. The depth was set to $0.3 \mathrm{~m}$ and the depth-averaged flow velocity was approximately $0.3 \mathrm{~m} / \mathrm{s}$. Comprehensive flow velocity measurements were taken laterally (cross-flume) and throughout the depth upstream of the wall split, and also in the sub channels on both sides of the wall. This velocity deficit would be able to give a measure of the extent to which the flow slowed down or accelerated due to the presence and various positions of actuator fences and is given by the expression:

$$
U_{\text {deficit }}=1-\frac{U_{w}}{U_{o}}
$$

Where $U_{\text {deficit }}$ is the velocity deficit, $U_{w}$ is the velocity at a specific point in the wake of the disk and $U_{0}$ is the natural freestream flow velocity at this specific point.

\subsubsection{Wake mapping of actuator fences}

An actuator fence with porosity (ratio of open to total area) of 0.38 was placed in the centre of one of the sub channels at a distance of $0.4 \mathrm{~m}$ downstream of the front of the split, which was also $8.9 \mathrm{~m}$ from the inlet of the flume. This setup is displayed graphically in Fig. 2. Flow velocity measurements were then taken in both sub channels. The first position to be measured was 3 fence diameters downstream of the fence and up to 21 diameters downstream of it in increments of 3 diameters. For each downstream position, several lateral positions 
were taken, while for each lateral position, 8 flow depths in increments of 30mm depth were taken. A similar analysis was carried out for the case of two actuator fences being present in a single sub channel. In this case two fences of porosity 0.38 and 0.4 were placed alongside each other. This case represented a very high blockage ratio, as the total width of the sub channel was approximately $635 \mathrm{~mm}$ and the width of the two fences combined was $600 \mathrm{~mm}$. Once again the wake was examined by taking flow velocity measurements at several positions downstream of the fences, in both channels, using the ADV.

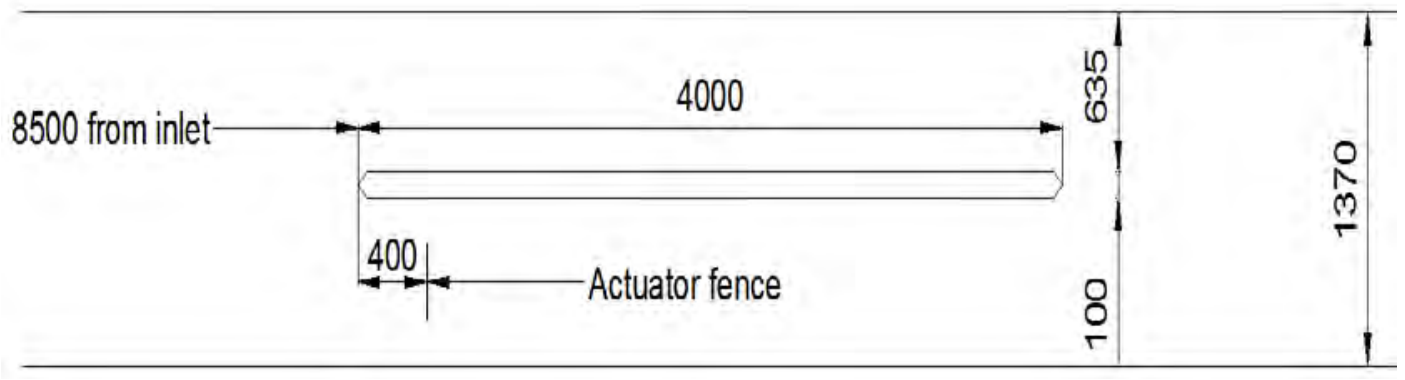

Fig.2. Plan view of Chilworth channel with porous actuator fence and split present (all dimensions in $\mathrm{mm}$ )

\subsubsection{Flow acceleration analysis}

As well as wake mapping in both channels, flow acceleration effects in the empty sub channel were examined. ADV's were positioned at two lateral points in the empty channel at a distance of $0.4 \mathrm{~m}$ from the front of the wall split, or $8.9 \mathrm{~m}$ from the inlet to the channel (Fig. 3). In the case of a sub channel with a single fence, a fence of porosity 0.4 was used. The fence was placed in 3 lateral positions, with the centre of the fence being positioned $180 \mathrm{~mm}$, $320 \mathrm{~mm}$ and $460 \mathrm{~mm}$ from the sidewall of the flume. For each of these lateral positions, the fence was also placed in the same downstream position as the front of the split, and was then moved gradually back in $100 \mathrm{~mm}$ increments to $500 \mathrm{~mm}$ downstream of the front of the split, then in $200 \mathrm{~mm}$ increments up to $2000 \mathrm{~mm}$ downstream. For each of these fence positions, the ADV recorded the flow velocity magnitude at the aforementioned points in the empty sub channel. Similar analysis was carried out for the case of a sub channel with two actuator fences present. Due to the fences occupying the vast majority of the width of the channel, changes to lateral position were not examined. Instead only the changes to the downstream positions were examined exactly as described for the case of a single fence. The high blockage ratio also meant that flow acceleration effects were anticipated.

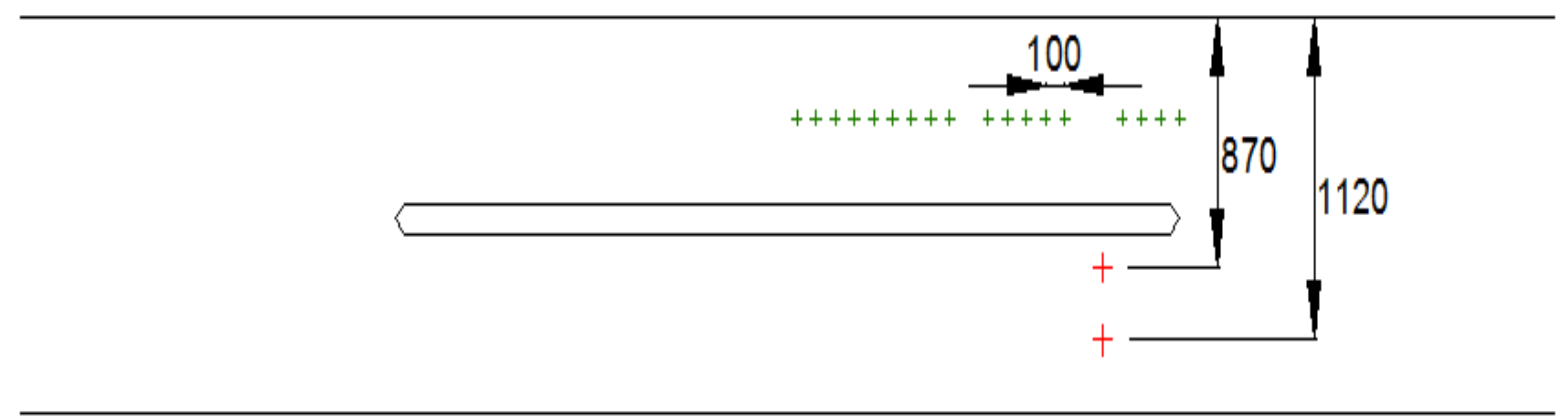

Fig.3: Diagram of Chilworth flume setup indicating location of split, measurement points in empty channel (indicated in red) and points where fence centre is located (indicated in green). 


\section{Results and discussion}

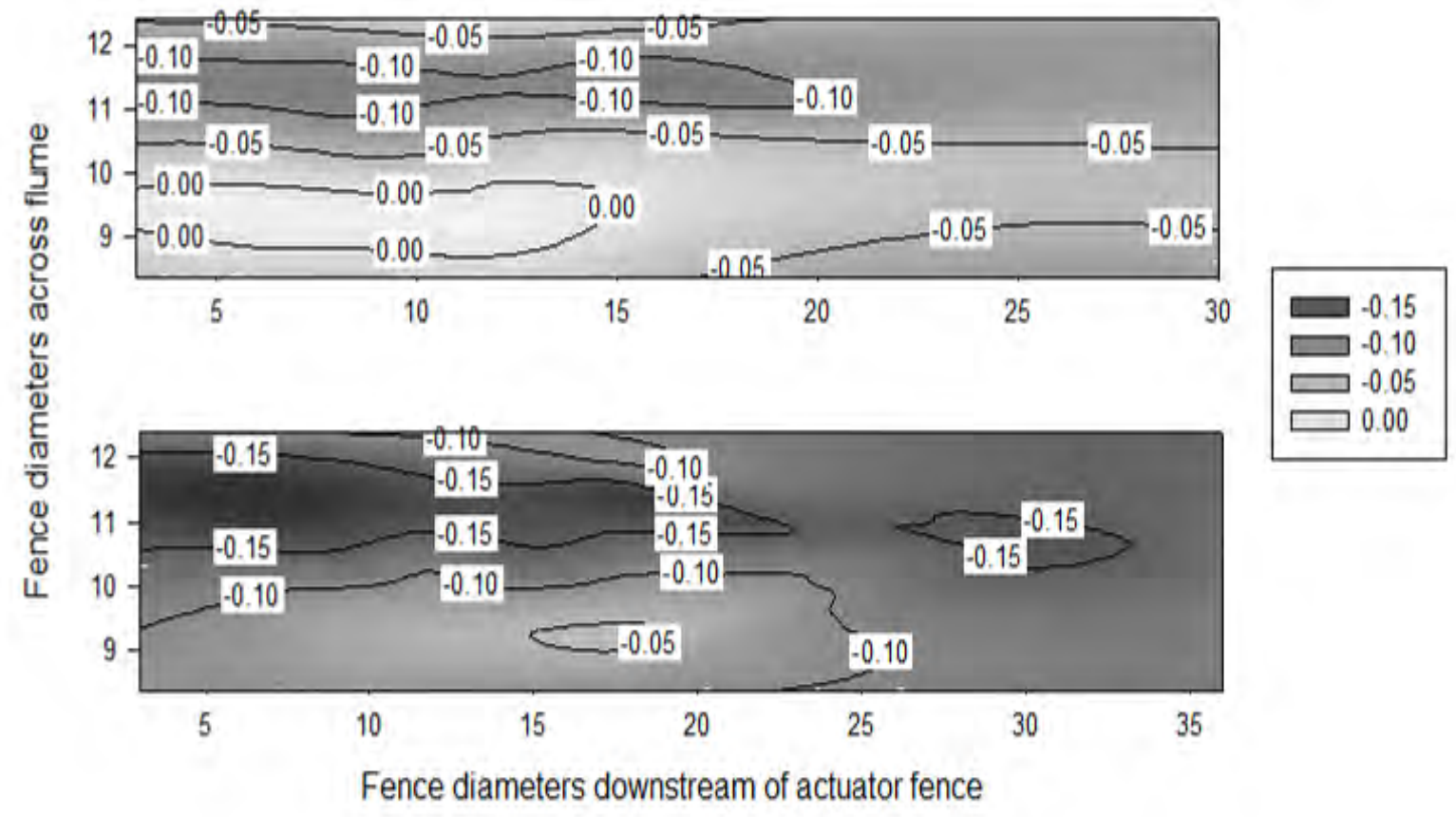

Fig.4. Contour plots showing velocity deficit variations in empty sub channel downstream of single 0.38 porosity fence (top) and downstream of 0.38 and 0.4 porosity fence (bottom).

Figure 4 shows the results of the wake mapping analysis of the areas downstream of both the single fence (top figure) and two fences (bottom figure), as discussed in section 3.4.2. These plots show that some flow acceleration is present in both cases, but also that it is much greater when two fences are present in the opposite channel. Velocity deficit values are zero or very close to zero in the top figure, while they approach values of -0.15 in the bottom figure.

Figures 5 and 6 show the results of the flow acceleration analysis outlined in section 3.4.3. These also demonstrate the presence of flow acceleration due to the presence of fences in the opposite channel. In the case of a single fence in figure 5 , there is no immediately apparent definite pattern or relationship between fence position, both laterally and in the downstream direction, and the percentage increase in freestream velocity magnitude. There is only a small difference between the readings given by the ADV positioned at $870 \mathrm{~mm}$ from the sidewall (left) and that positioned $1100 \mathrm{~mm}$ from the sidewall (right). Despite this lack of any definite relationship, it should be noted that the flow acceleration is quite small in itself, being $7 \%$ or less. Also the Chilworth flume has a variation in flow velocity at any point of approximately between $1 \%$ and $2 \%$ for any flow rate. For such small changes to flow, a lack of any definite relationship between the investigated parameters is not an unexpected result.

Much higher flow acceleration is observed from the results for the case of two actuator fences as displayed in figure 6 . The scatter plot shows flow acceleration of between $8 \%$ and $14 \%$. $14 \%$ flow acceleration is a potentially significant result from a tidal farm perspective. If the installation of more tidal converters in this empty channel could be justified both economically and environmentally, there is potential for up to $48 \%$ more power to be 

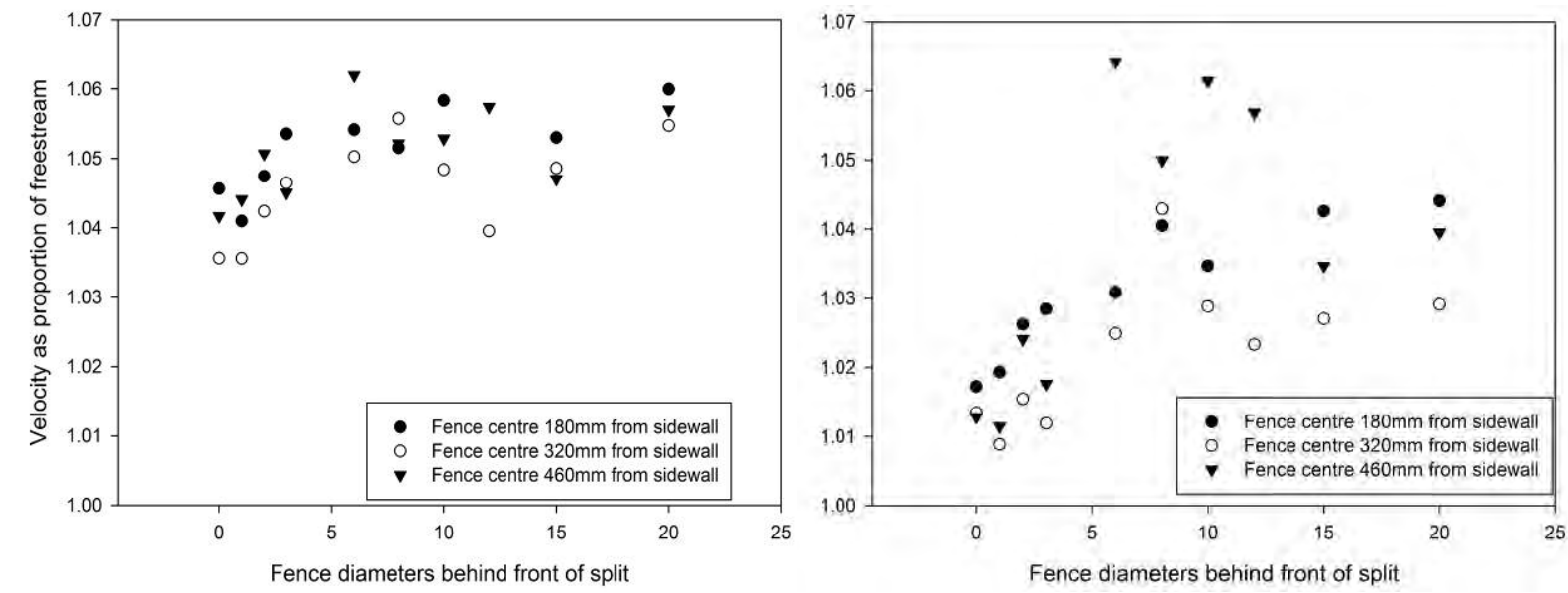

Fig.5. Flow acceleration in empty sub channel at lateral positions of $870 \mathrm{~mm}$ (left) and $1120 \mathrm{~mm}$ (right) due to movement of single actuator fence in opposite channel.
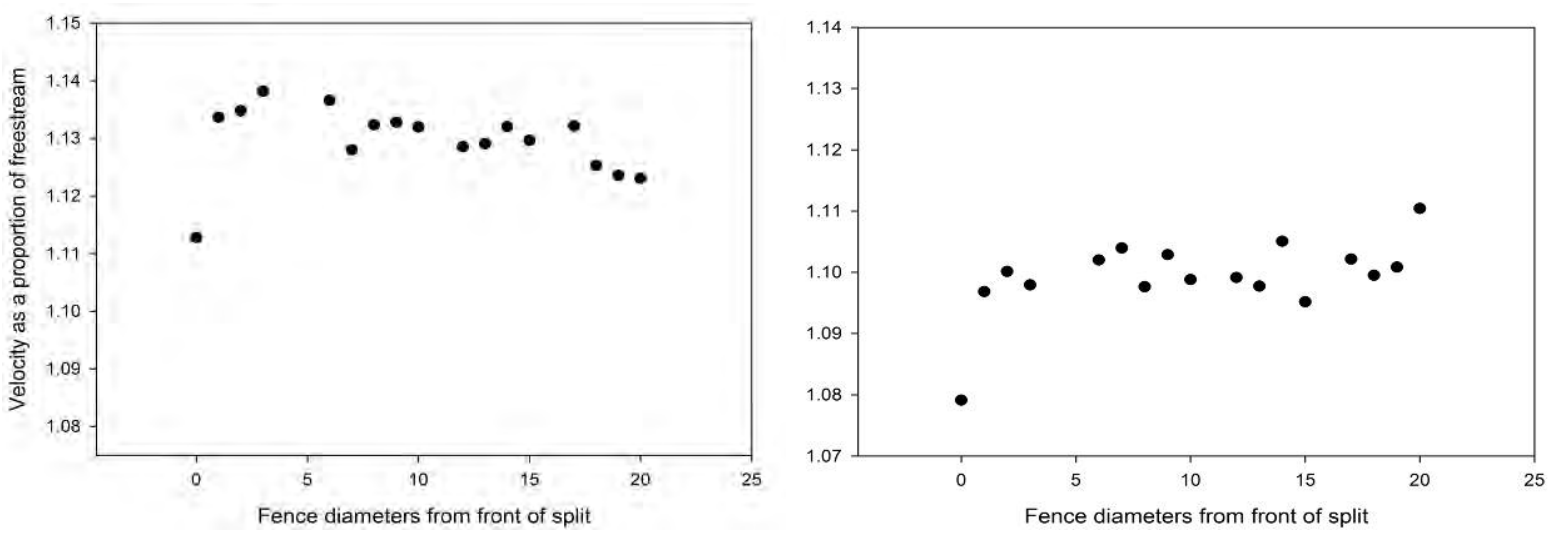

Fig.6. Flow acceleration in empty sub channel at lateral positions of $870 \mathrm{~mm}$ (left) and $1120 \mathrm{~mm}$ (right) due to movement of two side by side actuator fences in opposite channel.

extracted than without arrays in the other sub channel. However this would obviously be dependent on other factors such as internal turbine efficiency. It is also interesting to note from the scatter plots that the percentage acceleration is higher closer to the split than further laterally along the channel. This only occurs for the case of two fences. This might suggest that the presence of a greater number of turbines in an array may cause the flow to diverge further upstream than smaller arrays. Nearer to the split, there is also some reduction in flow acceleration as the array is moved further back from the split. This is another result which does not appear to happen further laterally across the channel. This may suggest that the flow is steadier and developed further away from the split, and that changes to longitudinal positioning of the array may only affect flow velocities in certain regions of the empty sub channel.

\section{Conclusions and future work}

The single actuator fence in this analysis occupied approximately $1 / 6^{\text {th }}$ of the total area of one sub channel $\left(1 / 3^{\text {rd }}\right.$ of the depth and $1 / 2$ of the width), and resulted in relatively low flow acceleration. There was also no obvious relationship between acceleration and either longitudinal or lateral position. In contrast, two actuator fences occupying $1 / 3^{\text {rd }}$ of the total area resulted in much higher acceleration, in some cases up to $14 \%$, with some dependence on longitudinal position apparent. These results suggest that total area blocked by turbines in a sub channel has implications for flow acceleration effects. Future work will examine this 
relationship between acceleration and blockage by using different sized actuator fences in one sub channel.

Higher flow acceleration in the region closer to the split also suggests that the initiation of flow divergence between the two sub channels may be dependent on the area blocked in one sub channel. Future work will examine the area upstream of the front of the tidal split in an attempt to determine at what point flow divergence begins and how dependent is the point of flow divergence on the area occupied by turbines. Results also show that high flow acceleration still occurs even when the actuator fences are a relatively large distance downstream of the front of the split. Future experimentation will attempt to determine at what array position downstream of the split flow acceleration will no longer exist.

\section{References}

[1] T. Daly, L.E. Myers, A.S. Bahaj, Experimental analysis of the local flow effects around single row tidal turbine arrays, Proceedings of the $3^{\text {rd }}$ International Conference on Ocean Energy, 2010.

[2] A. Mortimer, Tidal power with Hammerfest Strom technology: Towards commercialisation, Proceedings of 3rd International Conference on Ocean Energy, 2010.

[3] R.H. Karsten, J.M. McMillan, M.J. Lickley, R.D. Haynes, Assessment of the tidal current energy in the Minas Passage, Bay of Fundy, Proceedings of the Institution of Mechanical Engineers, Part A: Journal of Power and Energy 222, 5, pp. 493- 507

[4] B.L. Polagye, M. Kawase, P. Malte, In-stream tidal energy potential of Puget sound, Washington, Proceedings of the Institution of Mechanical Engineers, Part A: Journal of Power and Energy 223, 5, pp. 571- 587

[5] J.F. Atwater, G.A. Lawrence, Power potential of a split tidal channel, Renewable energy 35, 2, pp. 329-332.

[6] C. Garrett, P. Cummins, The power potential of tidal currents in channels, Proceedings of the Royal Society A: Mathematical, Physical and Engineering Sciences, 461, 2060, pp. 2563-2572.

[7] G. Sutherland, M. Foreman, C. Garrett, Tidal current energy assessment for Johnstone Strait, Vancouver Island, Proceedings of the Institution of Mechanical Engineers, Part A: Journal of Power and Energy 221, 2, pp. 147- 157

[8] B.L. Polagye, P.C. Malte, Far field dynamics of tidal energy extraction in channel networks, Renewable energy, 36, 1, 222-234.

[9] L.E. Myers, A.S. Bahaj, Experimental analysis of the flow field around horizontal axis tidal turbines by use of scale mesh disk rotor simulators, Ocean engineering, 37, 2-3, 218227.

[10] L. Cea, J. Puertas, L. Pena, Velocity measurements on highly turbulent free surface flow using ADV, Experiments in fluids 42, 3, pp. 333-348. 